\title{
Stress gradient analysis by noncomplanar x-ray diffraction and corresponding refraction correction
}

\section{Andrei Benediktovitch ${ }^{1, a}$, Tatjana Ulyanenkova ${ }^{2, b}$, Jozef Keckes ${ }^{3, c}$, and Alex Ulyanenkov ${ }^{2, \mathrm{~d}}$}

\author{
${ }^{1}$ Department of Theoretical Physics, Belarusian State University, Nezavisimosti Avenue 4, Minsk, \\ Belarus \\ ${ }^{2}$ Rigaku Europe SE, Am Hardtwald 11, Ettlingen, Germany \\ ${ }^{3}$ Department of Physics, The University of Leoben, Austria \\ aandrei.benediktovitch@atomicus.by, bTatjana.Ulyanenkova@rigaku.com, \\ cjozef.keckes@gmail.com, dAlex.Ulyanenkov@rigaku.com
}

Keywords: noncomplanar diffraction geometry, residual stress depth profiles, refraction and absorption, DWBA

\begin{abstract}
X-ray residual stress analysis is a widespread nondestructive technique to investigate the residual stress and residual stress gradient in thin films and protective coatings. In the present contribution we introduce a new method based on the noncomplanar measurement geometry that allow to span large area of $\sin ^{2} \psi$ and penetration depth values without sample inclination. The refraction correction and absorption is considered in details for the noncomplanar measurements. The proposed technique is applied to determine stress gradients of blasted hard TiN coatings.
\end{abstract}

\section{Introduction}

To investigate residual stresses nondestructively, X-ray diffraction (XRD) analysis based on the $\sin ^{2} \psi$ method (dependence of the deformations from the $\sin ^{2} \psi$ value [1]) is usually used. In the traditional $\sin ^{2} \psi$ plots each $\psi$ value corresponds to different penetration depth and in the case of thin layers with the presence of stress gradient resulting in deviation from linear behavior. From these nonlinearities one can in principle extract the information about stress gradient, see e.g. [2], however, due to interplay between various kinds of uncertainties the reliability of results is not high [3]. During the last decades a different modifications of this method appeared.

Using special set-up with help of so called scattering vector method [4] one may perform the stress measurements at fixed penetration depth, i.e. can obtain more reliable results. At synchrotron facilities there are a number of additional techniques available, e.g. energy-dispersive methods, through-surface strain scanning, see [5] and references there, or cross-sectional nano-diffraction [6], and other direct methods.

At the laboratory diffractometer the stress measurements $\left(\sin ^{2} \psi\right.$ scans) at fixed penetration depth can be done by varying at the same time three goniometer angles (incidence, $2 \theta$ and inclination angles) [7, 8]. This allows to obtain a series of $\sin ^{2} \psi$ plots each corresponding to a fixed information depth; from this dataset stress profile in Laplace space can be directly obtained. However, in parallel-beam experimental geometry it is not favorable to move to high inclination angles due to increased beam footprint effect. Also, in case of heavy samples or in-situ measurements involving various sample attachments (like high-temperature stress measurements [9]) it is not favorable to incline the sample stage. In this case one can achieve the same arrangement of sample normal and scattering vector by means of noncomplanar diffraction if the diffractometer is equipped with in-plane arm, like Rigaku SmartLab, Fig.1a.

The noncomplanar measurements utilizing in-plane arm enable to record complete pole figures in case of texture measurements [10], in case of residual stress measurements one can see that with the help of in-plane detector arm rotations and the combination of a several reflections it is possible to 


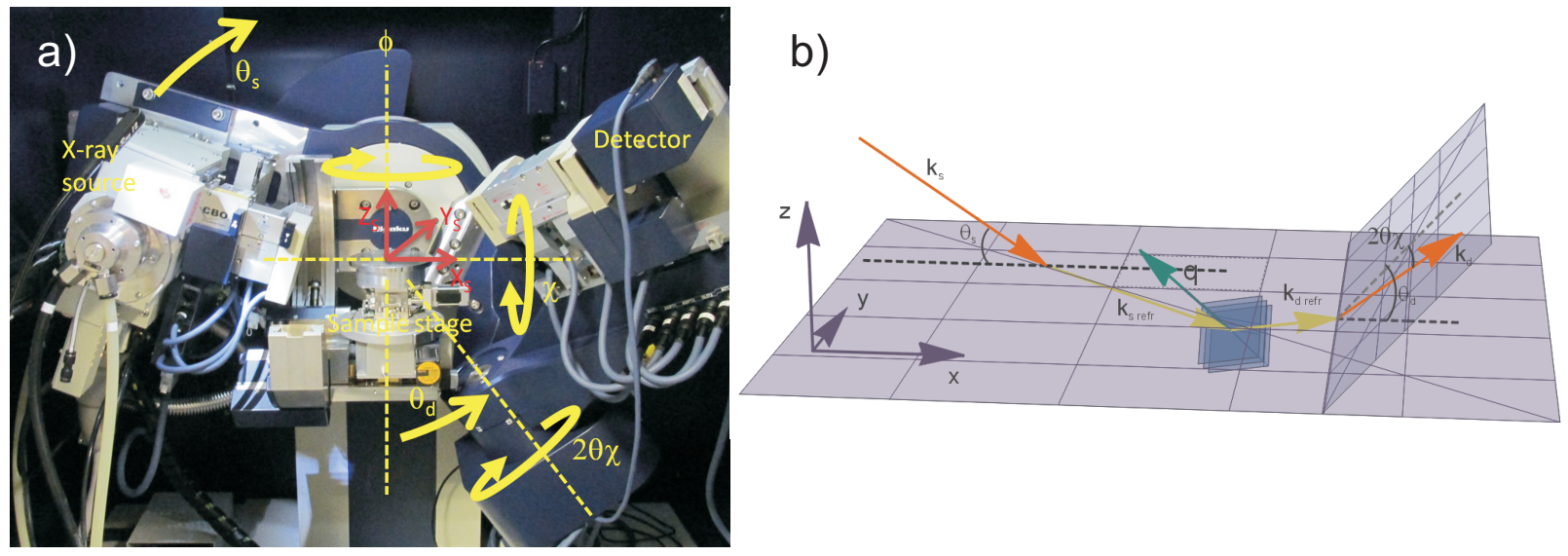

Fig. 1: a). In-plane diffractometer, where angle $\theta_{s}$ is the angle between the line connecting sample and x-ray source and the plane of the sample holder; angle $\theta_{d}$ is the angle between the axis of in-plane arm rotation and the plane of the sample holder (in case of no in-plane arm rotation $\theta_{d}$ is the angle between the line connecting sample and detector and the plane of the sample holder); angle $2 \theta \chi$ is the angle of the in-plane arm rotation; angle $\chi$ is the angle of the sample tilt against the plane of the sample holder; angle $\phi$ is the angle of the sample rotation around the sample normal. b). A sketch of refraction in noncomplanar case for zero sample tilt.

span large area of $\sin ^{2} \psi$ and penetration depth values even without sample inclination [11]. However, the correct analysis of the obtained data requires handling of bulky trigonometric expressions that replace simple relations familiar in the usual complanar case. The expressions for factors influencing peak positions [14] should be modified for noncomplanar case as well.

The residual stress analysis is sensitive to the precision of measured peak positions. The Lorentzpolarization, atomic scattering and absorption factors are important for wide $2 \theta$ scans involving number of Bragg peaks. For residual stress measurements only vicinity of a single Bragg peak is investigated, in this area the above mentioned factors are smooth functions and their influence on peak positions are minor [14]. The refraction correction cause systematic Bragg shift that is essential especially at grazing angles when is important to get information about the stress in surface layer. The dependence of refraction Bragg shift on $\psi$ angle can be interpreted as a fake residual stress and should be corrected for.

In the present contribution we give basic expressions for quantities of interest in noncomplanar case and discuss the refraction correction for this geometry. The conclusions derived are illustrated by depth resolved residual stress measurements of TiN hard coatings blasted by $\mathrm{Al}_{2} \mathrm{O}_{3}$ particles.

\section{Basic equations for noncomplanar $x$-ray residual stress analysis}

Below we will focus on diffractometer system depicted on Fig. 1a. It is characterized by angle $\theta_{s}$ defining the x-ray source position, by angles $\theta_{d}$ and $2 \theta \chi$ defining the detector position, and angles $\chi, \phi$ defining the sample tilt and rotation. In terms of these angles in the specimen reference frame the components of the wavevector $\mathbf{k}_{s}$ corresponding to the incoming wave are:

$$
k_{s}\left(\theta_{s}, \chi, \phi\right)=k_{0}\left(\sin \theta_{s} \sin \chi \sin \phi+\cos \theta_{s} \cos \phi, \cos \theta_{s} \sin \phi-\sin \theta_{s} \sin \chi \cos \phi,-\sin \theta_{s} \cos \chi\right),
$$

and the components of the wavevector $\mathbf{k}_{d}$ corresponding to the wave going from the sample to the detector are:

$$
\begin{aligned}
k_{d}\left(\theta_{d}, 2 \theta \chi, \chi, \phi\right)= & k_{0}\left(\cos \theta_{d} \cos 2 \theta \chi\right. \\
& \left.-\cos \chi \sin 2 \theta \chi+\cos 2 \theta \chi \sin \theta_{d} \sin \chi, \cos 2 \theta \chi \cos \chi \sin \theta_{d}+\sin 2 \theta \chi \sin \chi\right) .
\end{aligned}
$$


With help of these expressions one can determine the quantities needed for residual stress analysis. Namely, the Bragg angle is expressed as

$$
2 \theta_{B}=\arccos \left(\cos 2 \theta \chi \cos \left(\theta_{s}+\theta_{d}\right)\right)
$$

the $\psi$ angle between scattering vector and surface normal is

$$
\psi=\arccos \frac{\cos \chi\left(\cos 2 \theta \chi \sin \theta_{d}+\sin \theta_{s}\right)+\sin \theta_{s} \sin \chi}{\sqrt{2-2 \cos \left(\theta_{d}+\theta_{s}\right) \cos 2 \theta \chi}}
$$

and penetration depth (without refraction correction) is given as

$$
\tau=\frac{1}{\mu} \frac{\sin \alpha \sin \beta}{\sin \alpha+\sin \beta}, \quad \sin \alpha=\sin \theta_{s} \cos \chi, \quad \sin \beta=\cos 2 \theta \chi \cos \chi \sin \theta_{d}+\sin 2 \theta \chi \sin \chi .
$$

In the case of no sample tilt, $\chi=0$, for given Bragg angle, $\psi$ angle and penetration depth $\tau$ one can find corresponding source and detector positions defined by angles $\theta_{s}, \theta_{d}$ and $2 \theta \chi$ :

$$
\begin{array}{r}
\theta_{s}=\arcsin \left[\left(\cos \psi-\sqrt{\cos ^{2} \psi-2 \tau \mu \cos \psi \csc \theta_{B}}\right) \sin \theta_{B}\right], \\
\theta_{d}=\arccos \frac{1+P \sin \theta_{s}}{\sqrt{1+P^{2}+2 P \sin \theta_{s}}}, \quad 2 \theta \chi=\arccos \frac{\cos 2 \theta_{B}}{\cos \theta_{s}+\theta_{d}}, \\
P=\frac{\left(\cos \psi+\sqrt{\cos ^{2} \psi-2 \tau \mu \cos \psi \csc \theta_{B}}\right) \sin \theta_{B}}{\cos 2 \theta_{B}} .
\end{array}
$$

Based on Eq. (6) one can determine accessable regions of angle $\psi$ and penetration depth $\tau$ and set $\sin ^{2} \psi$ measurements at fixed penetration depth $\tau$. To expand the range of $\sin ^{2} \psi-\tau$ area one can combine several Bragg reflections. For more detailed analysis and experimental verification see [11].

\section{Refraction correction and absorption in noncomplanar geometry}

The refraction correction in complanar case was considered in a number of papers, see e.g. [12], [13], [14]. The expressions in [12], [14] in the notations of angles $\theta_{s}, \theta_{d}$ (and $2 \theta \chi=\chi=0$ ) (see Fig. 1b) are

$$
\Delta 2 \theta_{B}=-\delta\left(\cot \theta_{d}+\cot \theta_{s}+2 \tan \frac{\theta_{d}+\theta_{s}}{2}\right)
$$

here the refraction index is $n=1-\delta-i \beta$. This expression is approximate, as one can see that at $\theta_{s} \rightarrow 0$ or $\theta_{d} \rightarrow 0$ or at $\theta_{d}+\theta_{s} \rightarrow 180^{\circ}$ it goes to infinity. Expression that remains finite in the limits of small angles are given in [13] and were checked experimentally. Below we extent the derivation given in [13] to noncomplanar case. Figure 2 shows a drawing of refraction in noncomplanar case.

The basics of $\mathrm{x}$-ray residual stress analysis is the Bragg law that which gives connection between peak position and interplanar spacing. It is consequence of kinematical theory of x-ray diffraction, in which X-rays in matter are described by plane waves same as in vacuum [15]. In order to take into account the refraction one has to use Distorted Wave Born Approximation (DWBA), which treats the propagation of x-rays in averaged polycrystalline medium in exact way but treats scattering due to periodic part of polycrystalline matter polarizability in perturbative way [15]. Hence, as a first step we have to find the wave field in the polycrystalline matter neglecting its crystalline periodicity. Assuming that a plane wave with a wave vector $\mathbf{k}$ is impinging on a semiinfinite sample with surface normal $\mathbf{N}$ the wave vector in matter is found based on the conservation of wave vector component $\mathbf{k}_{\|}$ parallel to the sample surface (Snell law) and dispersion equation for wave in the matter [15]:

$$
\mathbf{k}_{r e f r}=\mathbf{k}_{\|} \pm \mathbf{N} \sqrt{k^{2} n^{2}-k_{\|}^{2}}
$$


here signs \pm correspond to transmitted and refracted waves in matter. Below we limit our discussion to transmitted wave only which is justified for semiinfinite media. In stratified media, however, at grazing angles there is also refracted wave with which would also contribute to scattering and would lead to appearance of a shifted peak. Naturally, such effects can be expected to be significant only at angles close to the angle of total external reflection, but it may be quite interesting to try to detect this splitting experimentally.

From Eq. (8) performing some algebra one can find that normal component of the refracted wavevector $k_{r e f r_{\perp}}$ is

$$
k_{r e f r_{\perp}}=\frac{\sqrt{\sqrt{4 \beta^{2}+\left(k_{\perp}^{2}-2 \delta\right)^{2}}+k_{\perp}^{2}-2 \delta}}{\sqrt{2}}+i \frac{\sqrt{\sqrt{4 \beta^{2}+\left(k_{\perp}^{2}-2 \delta\right)^{2}}-\left(k_{\perp}^{2}-2 \delta\right)}}{\sqrt{2}} .
$$

According to DWBA one has to consider incoming wave and "time reversed" outgoing wave [15]. As result the intensity is given as

$$
I \sim\left|\int d \mathbf{r} \operatorname{Exp}\left(i \operatorname{Re}\left(\mathbf{k}_{\mathbf{d r e f r}}-\mathbf{k}_{\text {srefr }}\right) \mathbf{r}\right) \operatorname{Exp}\left(-\operatorname{Im}\left(k_{d \operatorname{refr} \perp}+k_{s \mathrm{refr} \perp}\right) z\right) V_{H K L}(\mathbf{r})\right|^{2},
$$

here $V_{H K L}(\mathbf{r})$ is periodic part of crystallite scattering potential corresponding to reflex HKL. From Eq. (10) one can see that the real part transmitted wavevector in the medium is responsible for Bragg peak formation and imaginary part is responsible for absorption. The magnitude of transmitted wavevector is usually described by Bragg angle calculated for wavevector $k_{0}$ of the radiation in vacuum. Determined in this way, Bragg angle corresponding to Eq. (10) is

$$
2 \theta_{B \text { refr }}=\arccos \left(1-q_{\text {refr }}^{2} / 2 k_{0}\right), \mathbf{q}=\operatorname{Re}\left(\mathbf{k}_{\mathbf{d r e f r}}-\mathbf{k}_{\text {srefr }}\right) .
$$

Combining together Eqs. (11), (9), (8), (1), (2) after some algebra one obtain

$$
\begin{aligned}
& 2 \theta_{B \text { refr }}=\arccos \left(\cos 2 \theta \chi \cos \left(\theta_{s}+\theta_{d}\right)+\frac{1}{2}\left(\left(k_{s \perp}+k_{d \perp}\right)^{2}-\left(k_{s \perp \text { refr }}+k_{d \perp \text { refr }}\right)^{2}\right)\right), \\
& k_{s \perp}=\sin \left(\theta_{s}\right) \cos (\chi), \quad k_{d \perp}=\sin \left(\theta_{d}\right) \cos (\theta \chi) \cos (\chi)+\sin (\theta \chi) \sin (\chi),
\end{aligned}
$$

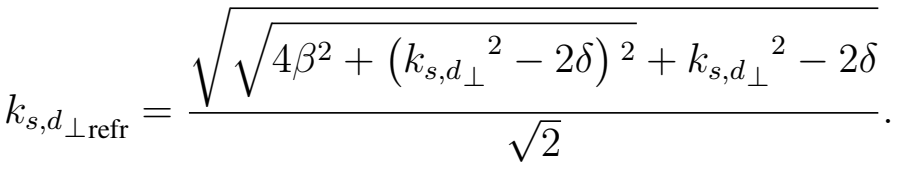

In the frame of current approach Eq. (12) is exact. It gives finite values at all angles including grazing case, where it takes into account refraction as well as at $2 \theta \rightarrow 180^{\circ}$ where it gives correction proportional to $\sqrt{\delta}$. Considering a practical case of nongrazing angles one can neglect the imaginary part $\beta$ of the refraction index and consider $\delta$ as a small quantity. Expanding Eq. (12) in series over $\delta$ one get

$$
\Delta 2 \theta_{B \text { refr }}=-\delta \frac{\left(2+\frac{k_{s \perp}}{k_{d \perp}}+\frac{k_{d \perp}}{k_{s \perp}}\right)}{\sqrt{1-\cos ^{2}\left(\theta_{d}+\theta_{s}\right) \cos ^{2} 2 \theta \chi}} .
$$

One can see that this expression has a crossover to Eq. (7) in complanar case. Figure 2a demonstrates this correction.

In the same way one can see that the penetration depth is given by

$$
\tau_{\text {refr }}=\frac{1}{2 \operatorname{Im}\left(k_{d \text { refr } \perp}+k_{\text {srefr } \perp}\right)}, \quad \operatorname{Im} k_{s, d_{\text {refr } \perp}}=k_{0} \frac{\sqrt{\sqrt{4 \beta^{2}+\left(k_{s, d_{\perp}^{2}}-2 \delta\right)^{2}}-\left(k_{s, d_{\perp}^{2}}-2 \delta\right)}}{\sqrt{2}},
$$

Performing the expanding in series over $\delta$ one comes to Eq. (5), here $\mu=2 k_{0} \beta$. Figure $2 \mathrm{~b}$ demonstrates this. In the same way one can find corrections to Eq. (4), however, they are much smaller than experimental uncertainties connected with peak position determination and can be neglected. 

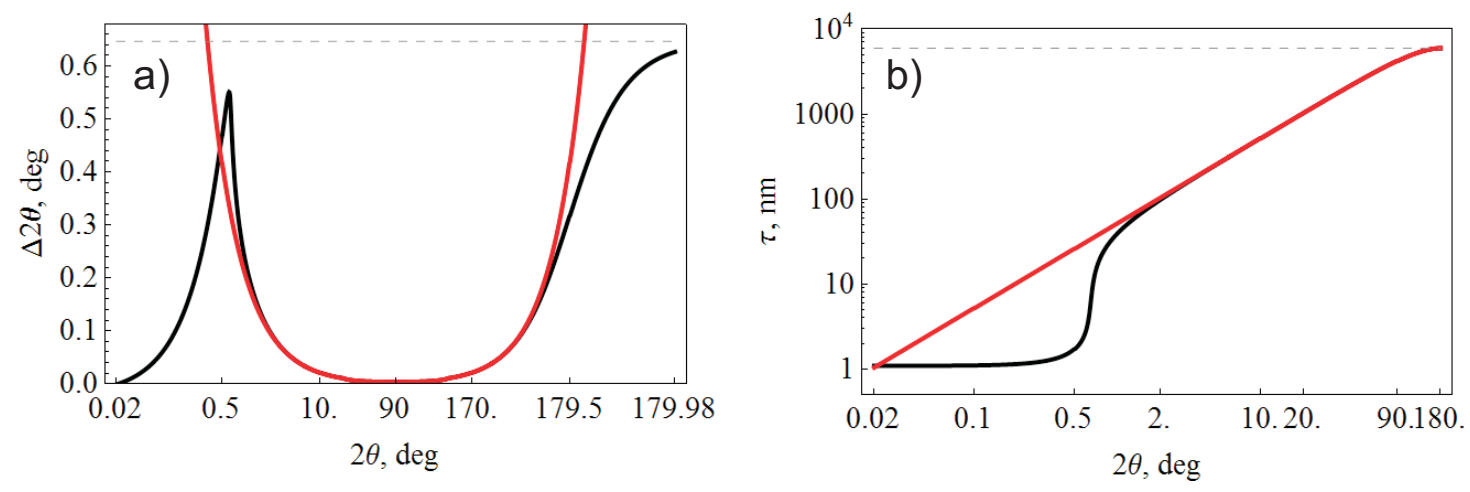

Fig. 2: a). The quantity $2 \theta-2 \theta_{B}$ according to Eq. (12) (black) and according to Eq. (5) (red) for conventional $\theta-\theta$ scan. Dashed line is limiting value of $2 \sqrt{2 \delta}$. Refraction index is taken for TiN. b). The penetration depth according to Eq. (13) (black) and according to Eq. (5) (red) for conventional $\theta-\theta$ scan. Dashed line is limiting value of $1 / 2 \mu$.

\section{Experimental details}

In order to check the approach of stress gradients measurements outlined above and evaluate importance of refraction corrections we have investigated residual stress state in the hard TiN coating. The polycrystalline TiN coating was deposited on WC/Co substrate using CVD at $900{ }^{\circ} \mathrm{C}$. The reactant gases were $\mathrm{N}_{2}, \mathrm{H}_{2}$, and $\mathrm{TiCl}_{4}$. The final coating thickness was $11.5 \mu \mathrm{m}$. The coating was wet blasted using a pressure equal to $4.0 \cdot 10^{5} \mathrm{~Pa}$. The diameter of the $\mathrm{Al}_{2} \mathrm{O}_{3}$ particles used for the blasting process was approximately $50 \mu \mathrm{m}$.

The XRD experiments were performed on a five-circle SmartLab diffractometer from Rigaku equipped with a $9 \mathrm{~kW}$ rotating anode using $\mathrm{Cu} \mathrm{K} \alpha$ radiation $(\lambda=1.54056 \stackrel{\circ}{\mathrm{O}})$. X-ray diffraction stress measurements were carried out in parallel-beam geometry using in-plane diffractometer arm rotation without sample tilt (see Fig. 1). The parallel beam optic was used to avoid any influence of the sample surface quality on the peak position. The diffractometer was carefully aligned and checked on the $\mathrm{LaB}_{6}$ standard sample. The diffractometer is equipped with cross-beam optic (CBO, patented Rigaku Corp.) collimating the incident beam and equatorial divergence of the diffracted beam is controlled by a $0.5^{\circ}$ PSA. For lateral collimation the $0.5^{\circ}$ in-plane PSC (parallel slit collimator - a set of vertical plates) were used.

In the used technique to perform $\mathrm{x}$-ray residual stress measurements with depth resolution the $2 \theta$ values should be varied close to Bragg angle to find peak position, this scan should be done at different $\sin ^{2} \psi$ values at fixed penetration depth. From the $\sin ^{2} \psi$ plots measured at various penetration depths the values of residual stress corresponding to different penetration depths might be found.

We used the $2 \theta$ scanning in 6 degree interval by changing $\theta_{s}$ and $\theta_{d}$ in equal steps. A step width on $2 \theta$ was chosen to be $\triangle 2 \theta=0.02^{\circ}$ and the rate during the measurements was equal to $0.02{ }^{\circ} \mathrm{min}^{-1}$. During such scans at non zero $2 \theta \chi$ angle the detector traverses the Debye ring at, in general, arbitrary angle to the Debye ring normal.

To perform the depth resolved measurements the peak positions were measured at 84 sets of initial $\theta_{s}, \theta_{d}, 2 \theta \chi$ angles, corresponding to points in $\sin ^{2} \psi-\tau \mu$ space. For the calculation of these points the set of TiN reflections with Bragg angles equal to 36.6, 42.0, 61.8, 93.17 and 104.68 were used. The angle values were calculated according to formalism above for the case of no sample tilt $(\chi=0)$.

The diffraction elastic constants $S_{1}^{h k l}$ and $1 / 2 S_{2}^{h k l}$ were calculated by means of the Neerfield-Hill model (arithmetical average of Reuss and Voight model). In the evaluation procedure, the stress state of the hard coatings was assumed to be biaxial.

It is known the texture affect the results of residual stress measurements. Pole figures for (111), (200) and (220) reflections were measured with the same in-plane setup. 

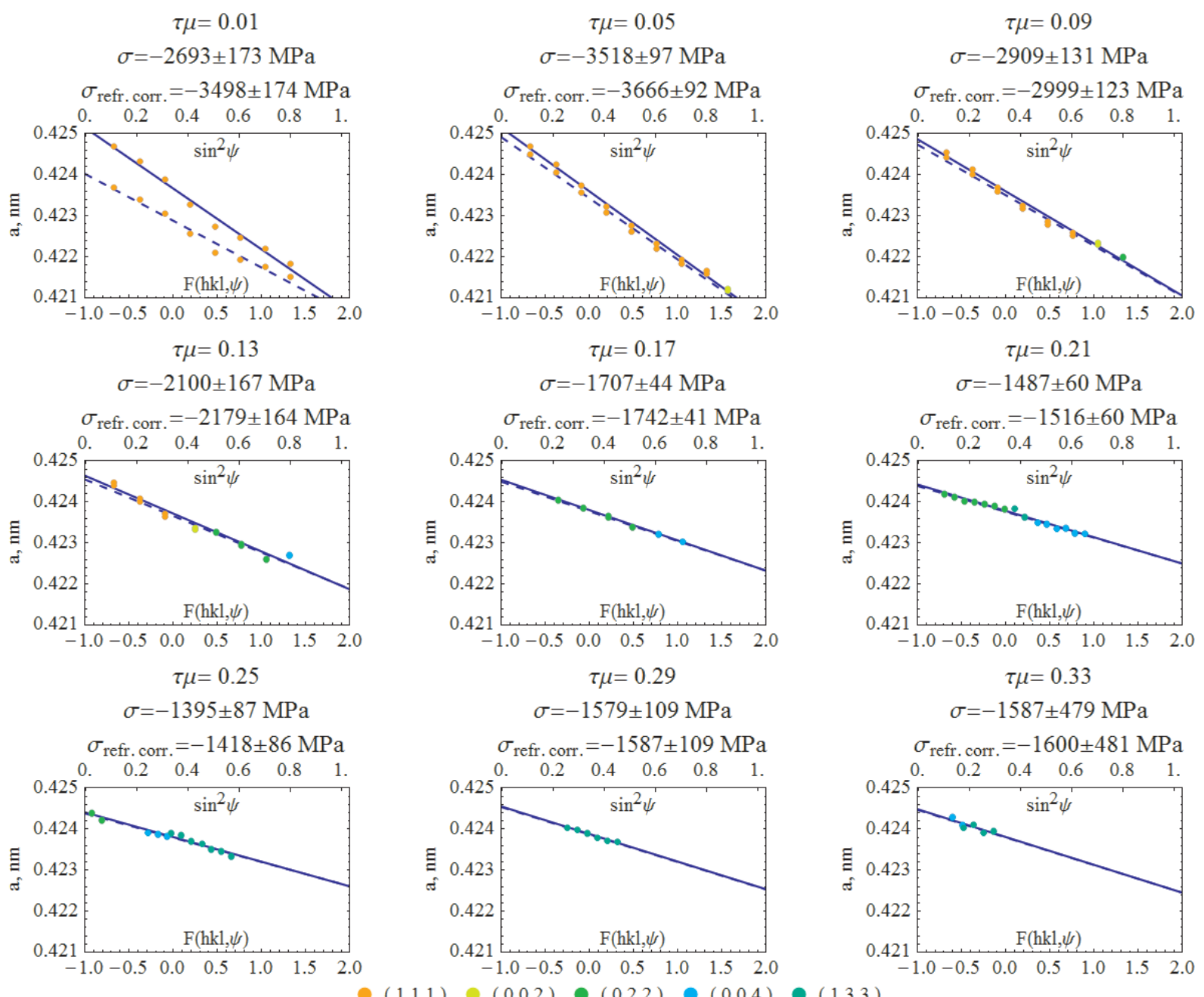

$\sigma=-1487 \pm 60 \mathrm{MPa}$

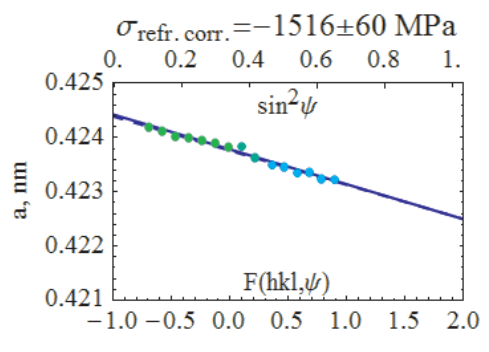

$\tau \mu=0.29$

$\sigma=-1579 \pm 109 \mathrm{MPa}$

$\tau \mu=0.33$

$\sigma=-1587 \pm 479 \mathrm{MPa}$

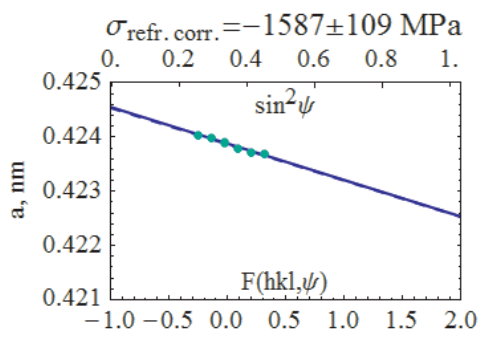

$\left(\begin{array}{llll}1 & 1 & 1\end{array}\right) \bullet\left(\begin{array}{llll}0 & 0 & 2\end{array}\right) \bullet\left(\begin{array}{lll}0 & 2 & 2\end{array}\right) \bullet\left(\begin{array}{lll}0 & 0 & 4\end{array}\right)$

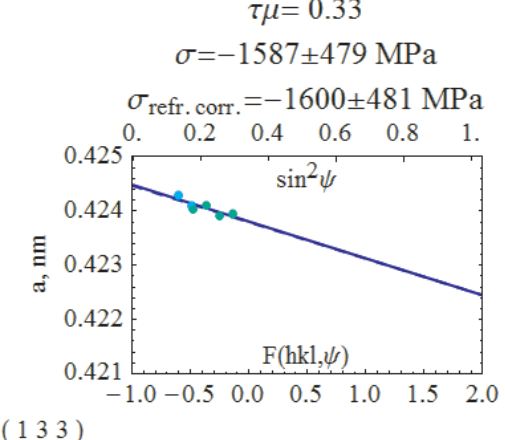

Fig. 3: Results of stress evaluation at different penetration depthes.

\section{Results and discussion}

The measured pole figures indicate the TiN thin films posses $\{110\}$ axial texture. The fraction of isotropic component was found to be about $70 \%$, the textured component was found to be quite broad. Together with Zener anisotropy ration close to 1 for TiN the texture effects can be neglected.

The dependencies between $a(h k l, \psi, \tau)$ and $\sin ^{2} \psi$ demonstrated on Fig. 3 show linear behavior. This plots demonstrate two set of data (before and after correction on refraction). Due to $\operatorname{small} \sin ^{2} \psi$ range covering the calculated stress values have large error bar. From the linear fit $\sigma(\tau)$ the stress values are obtained for fixed penetration depth $\tau$, see Fig. 4. One can see that for small penetration depth the refraction causes a "fake" stress that is comparable to actual stress in the sample. As it should be, the correction becomes negligible for larger penetration depth.

\section{Conclusion}

A method for residual stress profiling in the $\mu \mathrm{m}$ scale based on the X-ray diffractometer equipped with in-plane arm (like Rigaku SmartLab) is applied to characterize stress gradients in blasted TiN coatings. The in-plane arm usage was shown to increase the accessible region in $\sin ^{2} \psi-\tau$ space providing the $\sin ^{2} \psi$ plots in wider range, that improves the reliability of residual stress measurements. The effect of refraction at the sample surface which may cause the shift in Bragg peak position and therefore influences on the result of stress state determination is considered. It is demonstrated the 


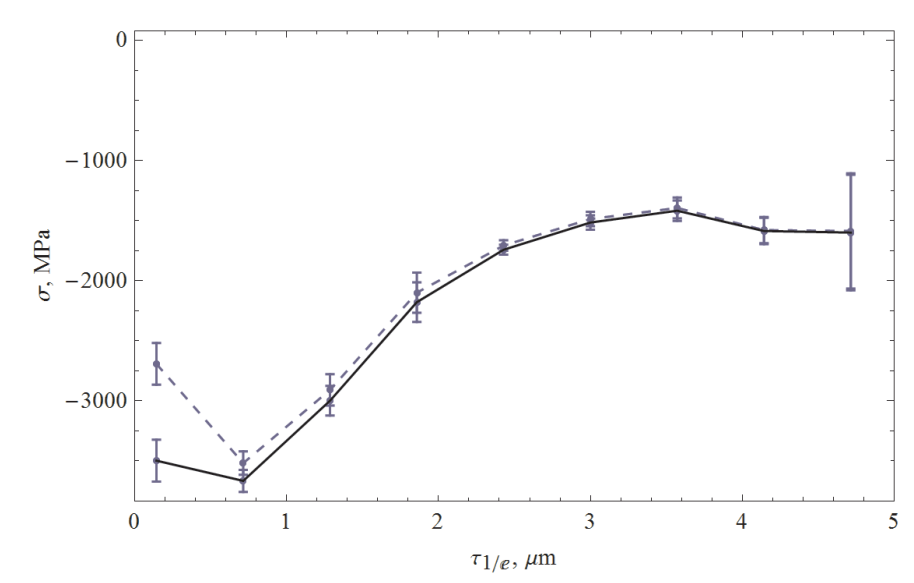

Fig. 4: Stress in laplace space without (blue line) and with (black line) refraction correction.

importance of accounting for the refraction effect in accurate measurements of lattice constant before stress calculations using small incidence angles.

\section{References}

[1] U. Welzel, J. Ligot, P. Lamparter, A. C. Vermeulen and E. J. Mittemeijer. J. Appl. Cryst. 2005. Vol. 38, pp. 1-29.

[2] H. Ruppersberg, I. Detemple, J. Krier. Physica Status Solidi (a). 1989. Vol. 116(2), 681687.

[3] H. Behnken and V.Hauk. Materials Science and Engineering: A. 2001. Vol. 300(1-2). pp. 41 51.

[4] C. Genzel. Physica Status Solidi (a). 1994. Vol. 146(2). pp. 629637.

[5] Ch. Genzel, I. A. Denks, M. Klaus, in Modern Diffraction Methods, Wiley-VCH, Weinheim. 2012, pp. 127-154.

[6] M. Stefenelli, J. Todt, A. Riedl, W. Ecker, T. Muller, R. Daniel, M. Burghammer, J.Keckes. J. Appl. Cryst. 2013. Vol. 46(5), pp.1378-1385.

[7] A. Kumar, U. Welzel and E. J. Mittemeijer. J. Appl. Cryst. 2006. Vol. 39, pp. 633-64638.

[8] Th. Erbacher, A. Wanner, T. Beck and O. Vhhringer. J. Appl. Cryst. 2008. Vol. 41, pp. 377-385.

[9] M. Bartosik, R. Pitonak and J. Keckes. Adv. Eng. Mat. 2011. Vol. 13, N 8, 705-711.

[10] K. Nagao, E. Kagami. Rigaku Journal. 2011. Vol. 27(2), 6-14.

[11] A. Benediktovitch, T.Ulyanenkova, J. Keckes, A. Ulyanenkov. JAC, sent.

[12] M. Hart, W. Parrish, M. Bellotto, G.S. Lim. Acta Cryst. 1988. Vol. A44, 193-197.

[13] M. F. Toney, S. Brennan. Phys. Rev. B. 1989. Vol. 39, 7963-7966.

[14] S.Wroński, K. Wierzbanowski, A. Baczmański, A. Lodini, Ch. Braham, W. Seiler. Powder Diffraction Suppl. 24 (S1) 2009 S11-S15.

[15] A. Benediktovitch, I. Feranchuk, A. Ulyanenkov, Theoretical Concepts of X-ray Nanoscale Analysis. Theory and Applications. Springer. 2014. 318p.

[16] A. Benediktovitch, H. Guerault, I. Feranchuk, V. Uglov, A. Ulyanenkov. Material Sci. Forum. 2011, 681, 121-126. 